\title{
Expanding Knowledge About Implementation of Pre-exposure Prophylaxis (PrEP): A Methodological Review
}

\author{
Rogério M. Pinto ${ }^{1}$ (D) Ashley Lacombe-Duncan ${ }^{1} \cdot$ Emma Sophia Kay $^{1} \cdot$ Kathryn R. Berringer $^{1,2}$
}

Published online: 10 July 2019

(c) The Author(s) 2019

\begin{abstract}
Methodological limitations in PrEP implementation studies may explain why PrEP implementation is lagging. This methodological review provides a description and critique of the methods used to identify barriers to PrEP implementation in the United States (2007-18). For each selected article, we provide: (1) research questions; (2) measures; (3) design; (4) sample (size and type); and (5) theoretical orientation. Among 79 articles which identified knowledge, attitudes, and behavioral and social/structural barriers to PrEP implementation, 51 (65\%) were quantitative; $25(32 \%)$ qualitative; and $3(4 \%)$ were mixedmethods; overall, just one-half described a conceptual approach. About two-thirds of articles were conducted with patients and one-third with healthcare providers. Our review reveals a paucity of longitudinal, mixed-methods, and ethnographic/ observational research and guiding theoretical frameworks; thus, the applicability of results are limited. We recommend that interventions aimed at PrEP implementation address barriers situated at multiple ecological domains, and thus improve PrEP access, uptake, and adherence.
\end{abstract}

Keywords PrEP implementation methods · PrEP methodological review $\cdot$ HIV prevention · Continuum of care

\section{Introduction}

At the end of 2015, the United States Centers for Disease Control and Prevention (CDC) estimated that 1,122,900 persons in the United States (US) were living with HIV; of these, 38,500 new infections occurred in 2015 alone [1]. In 2012, the US Food and Drug Administration (FDA) approved HIV pre-exposure prophylaxis (PrEP) - Truvada $^{\mathrm{TM}}$ [Emtricitabine/Tenofovir Disoproxil Fumarate (TDF/FTC)] — as a daily dosing strategy to reduce the risk of HIV infection for people most exposed. Research shows a risk reduction by $73 \%$ among adult men who have sex with men (MSM) and transgender women who take PrEP 90\% of the time [2]; and even greater efficacy (up to 99\%) for people with higher rates of PrEP adherence [3, 4]. Though PrEP reduces risk for HIV infection, only 56,600 Latino, African American, and White people are estimated to be

Rogério M. Pinto

ropinto@umich.edu

1 University of Michigan, School of Social Work, Office 2850, 1080 South University, Ann Arbor, MI 48109, USA

2 University of Michigan, Anthropology, Ann Arbor, MI, USA taking PrEP; even though an estimated 1.1 million people from these groups may benefit based on CDC clinical guidelines - this amounts to only about $5 \%$ of all people in the US who could benefit from PrEP taking it $[5,6]$.

In a comprehensive review of the literature, our team identified 30 barriers to PrEP implementation-steps patients and health providers must take in order to navigate healthcare systems and to ascertain access, delivery, and adherence to PrEP [7]. As from our original review, herein, the steps patients and providers must take to follow policies governing access to PrEP and to navigate healthcare systems are referred to as "PrEP implementation." Our conceptualization reflects the definition of implementation research as the "study of processes and strategies that move, or integrate, evidence-based effective treatments [in this case PrEP] into routine use, in usual care settings." ([8], p. 27) The review included research, between 2007 and 2017, in the fields of medicine, nursing, social work, and public health. We identified barriers across four ecological domains individual (patient), relationship (patient-service provider), community, and policy [9]. Among cognitive barriers, those affecting patients and providers included lack of knowledge about, and negative attitudes toward PrEP. Healthcare-level barriers included lack of communication about, funding for, and 
access to PrEP. The "purview paradox" was a key barrierHIV specialists trained to provide PrEP often do not tend to HIV-negative patients, while primary care physicians, who often see uninfected patients, are often not trained to provide PrEP. PrEP stigma and HIV stigma, transphobia and homophobia, sexism, and racism are also major barriers to PrEP implementation, contributing to disparities across sexual orientation, gender identity, and racial/ethnic background.

In order to decrease the rate of HIV infection, interventions to scale up PrEP will need to address identified barriers at multiple ecological levels. However, in the past decade, interventions that have been proposed to break PrEP implementation barriers have often been limited to one ecological level or another (e.g., individual or community). The failure to consider interventions targeting multiple ecological levels simultaneously may explain partly why PrEP implementation is lagging [7]. However, this failure may also be due to methodological limitations of PrEP implementation studies, as evidenced in our systematic review where we saw that studies tended to focus on one level (e.g., patient-level) while making recommendations on another (e.g., providerlevel) without supporting data [7]. We also found few published intervention studies to guide implementation, perhaps indicative of timing (e.g., early in PrEP implementation). Based on our systematic review of the PrEP implementation literature, we hypothesize that the methods used thus far have also been limited in several ways beyond sample characteristics (e.g. patient versus provider) and study design (e.g., exploratory versus intervention). Questions remain about the extent to which the methods used to identify barriers to PrEP implementation have progressed since PrEP became a major HIV prevention strategy and how research can be conducted to better inform advancements in PrEP implementation science.

Therefore, we have conducted an evaluation of the methods used thus far to identify barriers to PrEP implementation. We organized the current methodological review chronologically, and, for each article reviewed, we provide a summary of: (1) key elements of research questions; (2) measures; (3) research design; (4) sample (size and type); and (5) theoretical orientation. We describe how methods to study PrEP implementation have evolved over time, and we make recommendations about how to build on these methods to better capture PrEP implementation barriers and corresponding solutions as we move forward.

\section{Methodological Review: Conceptual Approach}

This methodological review is grounded in Whittemore et al. [10] model for integrative reviews and Munn et al. [11] typology/guidance for systematic reviews in the medical and health sciences. The integrative model and the typology guided us in our choice of a specific and clear set of inclusion/exclusion criteria for selection of articles, followed by a comprehensive search of published articles within a welldefined time period.

This methodological review builds on a systematic review that we published in 2018, and which included an examination of barriers to PrEP implementation published between 2007 and 2017 [7]. The current methodological review extends the original one to include 79 articles published between 2007 and 2018. The key goal of the original review was to identify barriers to PrEP implementation in the US. We were guided by a socioecological perspective [9] suggesting that barriers to PrEP implementation reside within different domains of reference: Individual and Relationships (patients and care providers); and Community and Policy Domains (policies governing HIV-prevention efforts, and both healthcare systems and agency settings guidelines). This approach in the context of PrEP implementation recognizes the roles of both patients and healthcare providers embedded within healthcare systems of all sizes who are required to follow multiple policies and guidelines [12]. In this case, these policies and guidelines refer to those regarding PrEP implementation-steps to navigate healthcare systems and which facilitate patient access and adherence to PrEP. By maintaining a socioecological approach to this methodological review, we continue to attend to the ways in which PrEP study methods address the holistic contexts within which PrEP implementation occurs.

\section{Methods}

\section{Procedures for Article Selection: Inclusion and Exclusion Criteria}

For the current methodological review, we updated the time period (2007-2017) we used for the original systematic review in order to include articles published in 2018. For the present methodological review, we changed inclusion and exclusion criteria slightly so as to include only articles containing a clear description of methods. In summary, we selected articles published between January 2007 and December 2018, a time period that included the development of the HIV continuum of care and high-impact prevention approach (treatment as prevention), the surge of evidence of PrEP effectiveness from large-scale clinical trials $[2,13,14]$, the subsequent FDA approval of PrEP for service settings [15], and, more recently, the confirmation that daily PrEP use is safe [16, 17].

We used the University of Michigan's ArticlesPlus, a comprehensive database of peer-reviewed clinical and academic journals in medicine, public health, social work, 
nursing, pharmacy, and law, to conduct our literature search. Our combination of search terms, including truncation operators (*) as follows:

Subject Terms: (HIV OR HIV/AIDS OR AIDS) AND Title: (PrEP OR "Pre-Exposure Prophylaxis") OR [(antiretroviral* OR pharmaceutical*) AND prevent*)] AND All Fields: [(worker* OR practitioner* OR provider*) AND (linkage* OR linking OR referral* OR implementation OR uptake)].

Our initial search (January 2007-June 2018) yielded 196 articles that [1] described implementation of PrEP programs for HIV prevention, and [2] focused on HIV service providers, medical, and social and public health service providers in agency settings in the US, and patients. We focused exclusively on the US because PrEP-related implementation policies and practices may differ profoundly across the globe. The inclusion criteria used to search articles published in 2018 were the same used for our published systematic review about barriers to PrEP implementation [7].

In order to include articles published through the end of the 2018, we attempted to update our search in January 2019. Unfortunately, in the interim the database system, ArticlesPlus, on which we had conducted our original search was discontinued by the University of Michigan. Therefore, we conducted our updated search using the new University of Michigan search interface, which consolidated ArticlesPlus with other library search interfaces. In order to meet the parameters of this new search tool, our search terms needed to be altered slightly (to limit operators and parenthetical clauses included within search term categories). The following updated search was conducted on January 13, 2019:

[subject:HIV OR HIV/AIDS OR AIDS AND title:PrEP OR "Pre-Exposure Prophylaxis" AND all_ fields:linkage* OR linking OR referral* OR implementation OR uptake] OR [subject:HIV OR HIV/ AIDS OR AIDS AND title:antiretroviral* OR pharmaceutical* AND prevent* AND all_fields:linkage* OR linking OR referral* OR implementation OR uptake]

Compared to our original search, which yielded 196 articles between 2007 and June 2018, this consolidated search tool yielded many more results. The search generated 340 articles published between 2007 and 2018, with 144 articles published in 2018 alone. We conducted a preliminary review based on titles and abstracts, eliminating 217 articles that did not meet inclusion criteria. The remaining 123 articles were then subjected to a full-text review, at which point we were able to eliminate an additional 44 articles that did not meet inclusion criteria. The final selection (2007-2018) included 79 articles. We provide a summary of procedures for article selection in Fig. 1.
Fig. 1 Article selection and inclusion/exclusion criteria

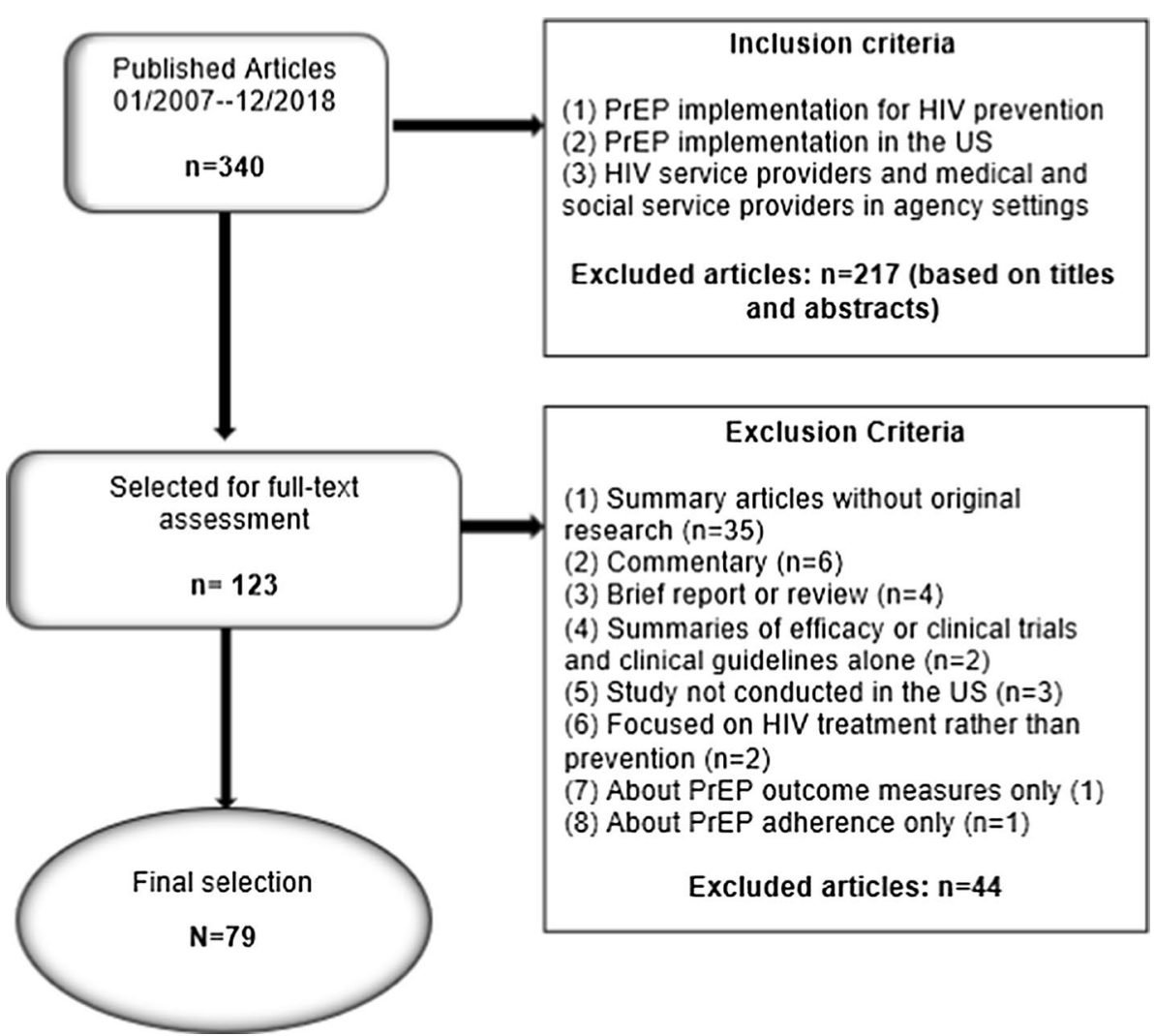




\section{Summary of Exclusions}

Our methodological review excluded the following types of articles: (1) summary articles without original research (e.g., systematic reviews, such as our own), (2) summaries of efficacy or clinical trials and clinical guidelines alone, (3) analysis of PrEP awareness and attitudes alone, (4) studies conducted outside of the US, (4) editorials and articles that included only a description of PrEP studies, (5) studies focused on HIV treatment rather than prevention, (6) studies about PrEP outcome measures only, (7) studies about PrEP adherence only, (8) models to determine PrEP eligibility alone, (9) epidemiological reports on PrEP and statistics alone, (10) cost-effectiveness studies alone, or (11) modelling studies alone.

\section{Data Extraction and Analysis}

To organize and manage our library, we created an Excel spreadsheet to record key information about each publication: title; authors; journal; publication date; journal type; theoretical approach; methodological approach (i.e., qualitative, quantitative, or mixed methods); and a summary of findings.

Our analysis focused on selecting articles that identified barriers to PrEP implementation in various ecological domains and which also reflected the inclusion criteria described above. We followed the same procedures that were used in the original systematic review (for details, see [7]). In summary, to enhance rigor to the analysis, we adopted a purposive sampling strategy-explicit search terms, inclusion and exclusion criteria, and procedures for article selection [18]. We borrowed basic principles of grounded theory as we selected the final set of articles for analysis grounded in our experiences as HIV researchers and practitioners in community settings [19]. We also brought different expertise in social work and anthropology. The authors held weekly 60-min discussions to finalize the list of articles contained myriad barriers to PrEP implementation and came to $100 \%$ agreement about which articles should be included and excluded from this review.

In order to examine the methods in each article, we organized articles by type of methods used-quantitative (Table 1), qualitative (Table 2), and mixed methods approach (Table 3). For each article, we identified: (1) key elements of research questions; (2) research design; (3) sample size and type; (4) underlying theoretical approach; and (5) key measures. The authors worked individually to examine the articles, and we met six times, for meetings lasting 1-2 h, to discuss the articles and to determine the classifications based on method type and key methodological elements. During the discussions, we also decided by consensus how to present the results.

\section{Results}

We included a total of 79 articles in this methodological review: [20-98] 51 (65\%) quantitative (Table 1) [20-70]; 25 (32\%) qualitative (Table 2) [71-95]; and three (4\%) mixedmethods (Table 3) [96-98]. Total of percentages exceeds 100 due to rounding up.

\section{Key Elements of Research Questions and Research Designs}

Reflecting our rigorous inclusion/exclusion selection criteria, all articles, regardless of method type, aimed to identify barriers to PrEP implementation. However, some studies had interrelated additional research questions. For example, several qualitative studies not only identified barriers, but described them [77, 84]. Other studies described the processes by which some patients may access PrEP by overcoming identified barriers $[88,95]$. Others focused on exploring structural issues that impact specific groups of people confronting disparaging socioeconomic problems (e.g., racism, homophobia, stigma, etc.), the combination of which deter great numbers of people from accessing PrEP [83]. Research questions focused on: (1) patient and service provider preferred modes of PrEP delivery, (2) patient engagement with PrEP including experiences of discontinuation, (3) patient and provider knowledge about and/or attitudes toward recommending and/or prescribing PrEP, (4) behavioral and psychosocial factors influencing PrEP access, implementation, and adherence, and (5) structural disparities (e.g., race, gender, stigma, etc.) in PrEP access and uptake.

\section{Key Measures}

Measures most commonly used evaluated cognitive factors such as PrEP knowledge, attitudes and concerns; a smaller proportion explored perceived barriers to access, utilization, and adherence; and some explored behavioral (e.g., risk compensation) and social/structural (e.g., stigma) factors. The most recently published studies (2018 onwards) explored more complex PrEP decision-making and uptake. For example, reasons for PrEP discontinuation, PrEP-related clinical decision-making, interprofessional collaboration, training, and provision of PrEP psychoeducation, and the role of local health departments in PrEP implementation were explored quantitatively, data of which may lend itself more readily to informing intervention development.

Quantitative articles mostly used cross-sectional surveys $(n=35 / 51,68 \%)$. Other quantitative articles used retrospective chart review, secondary analysis of cross-sectional population-based survey data, intervention study data, 


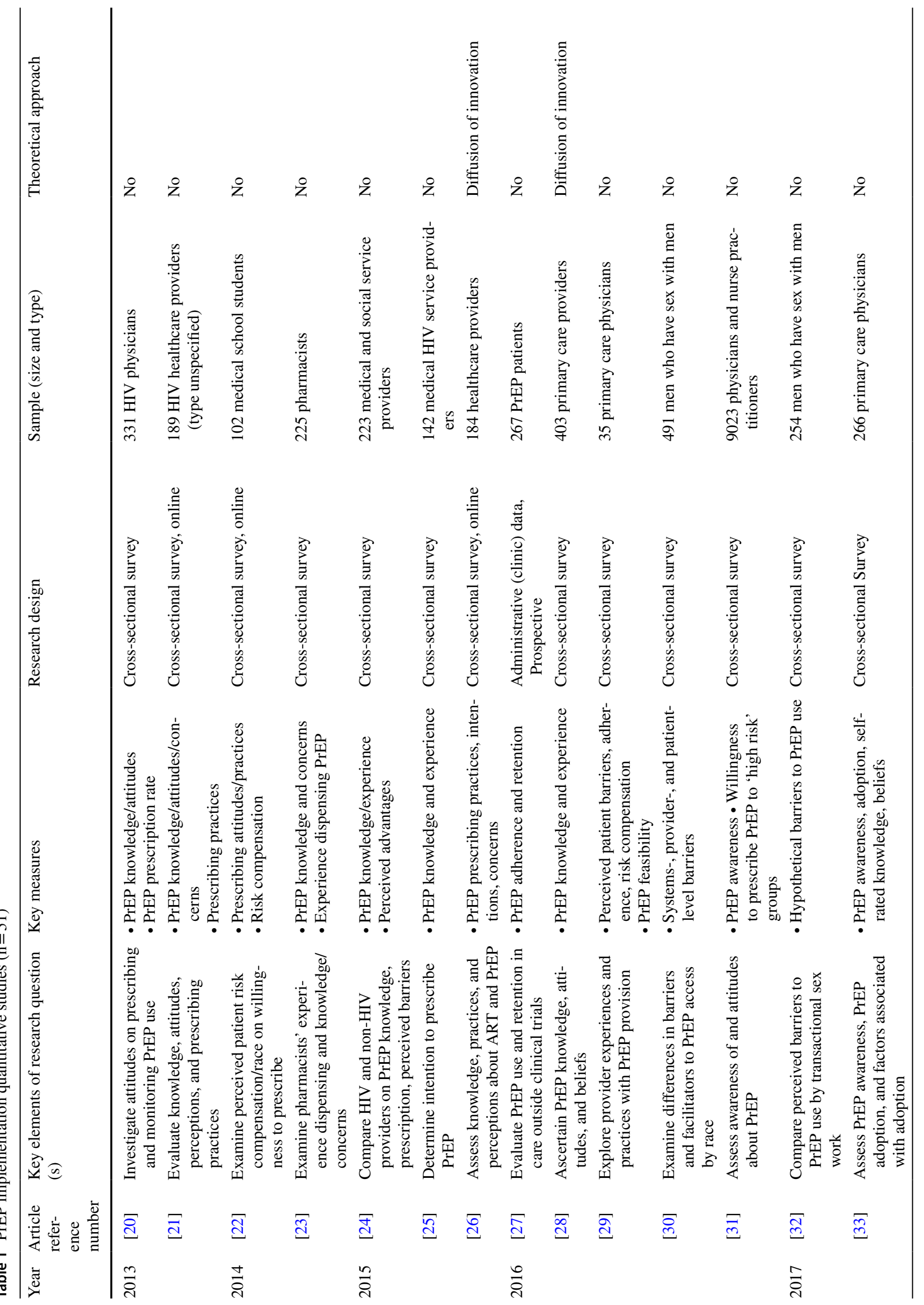




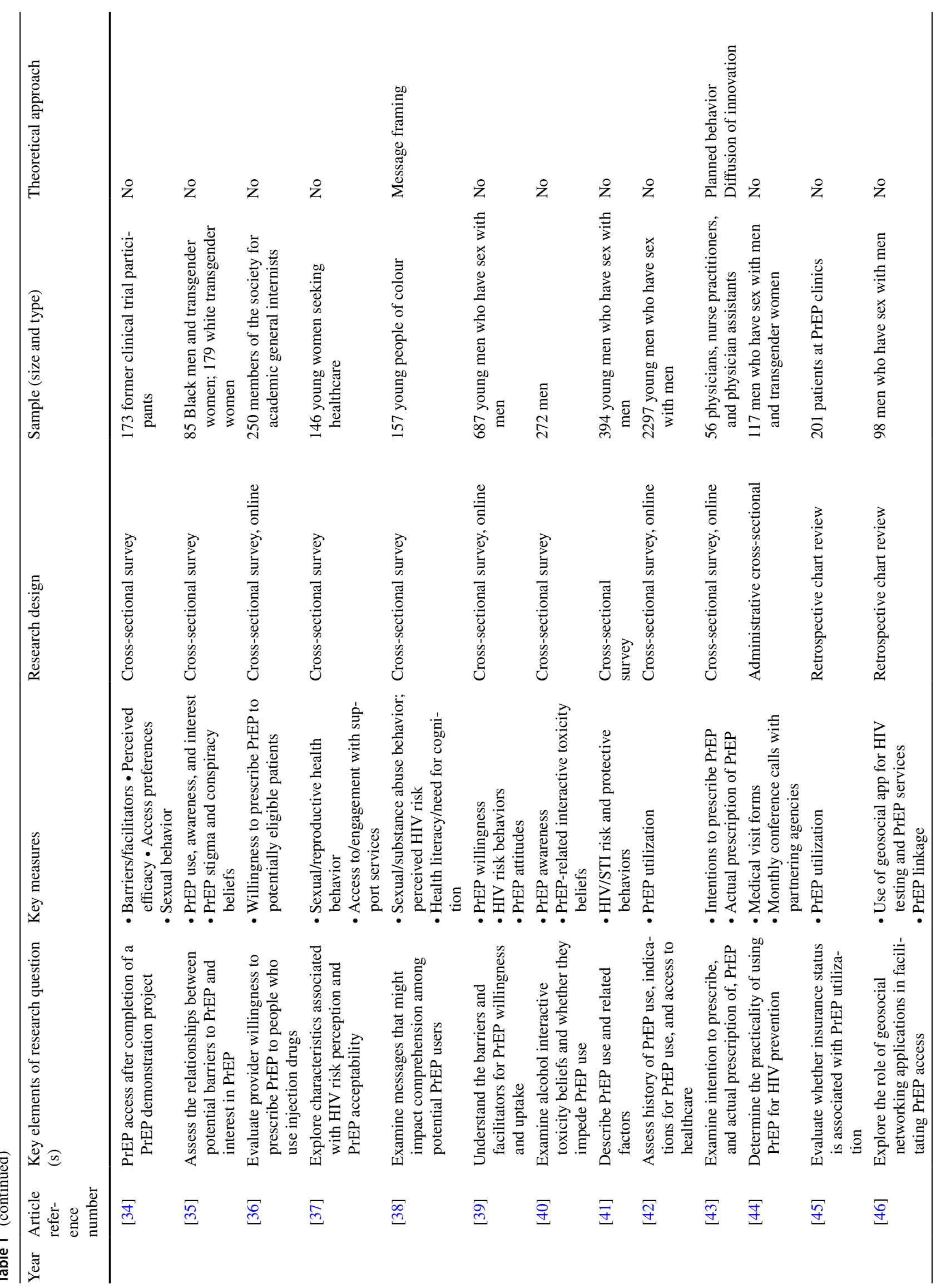




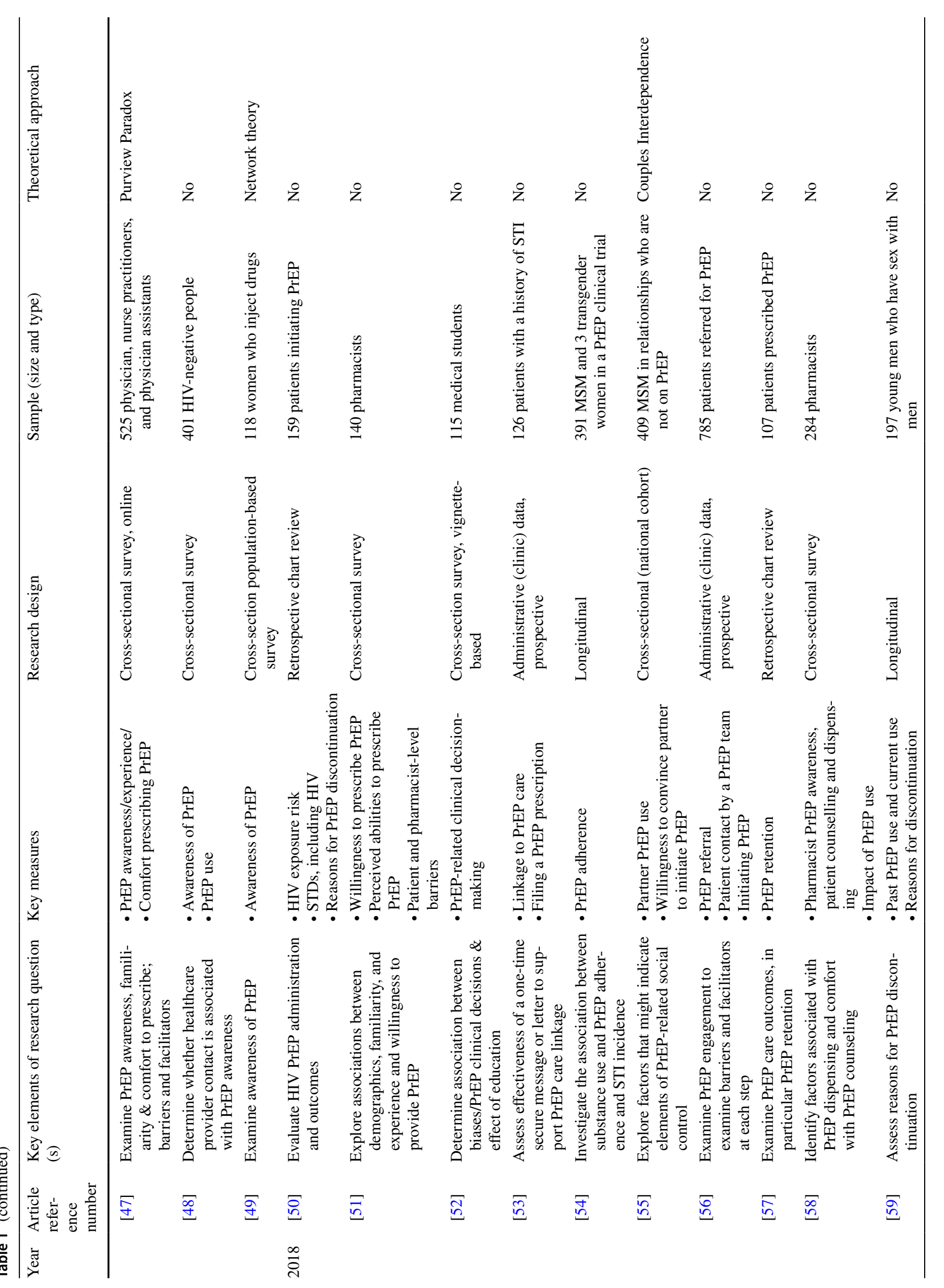




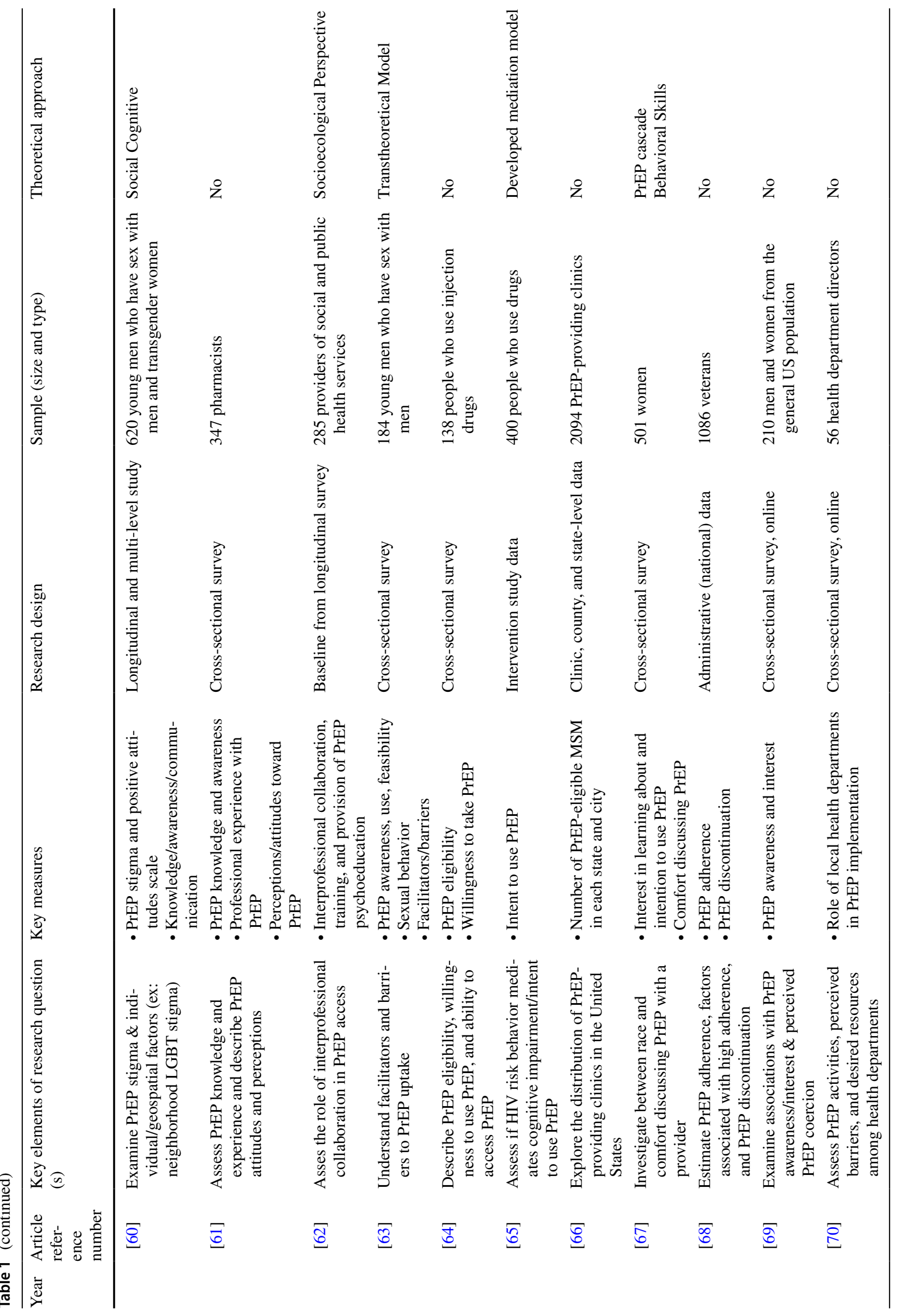




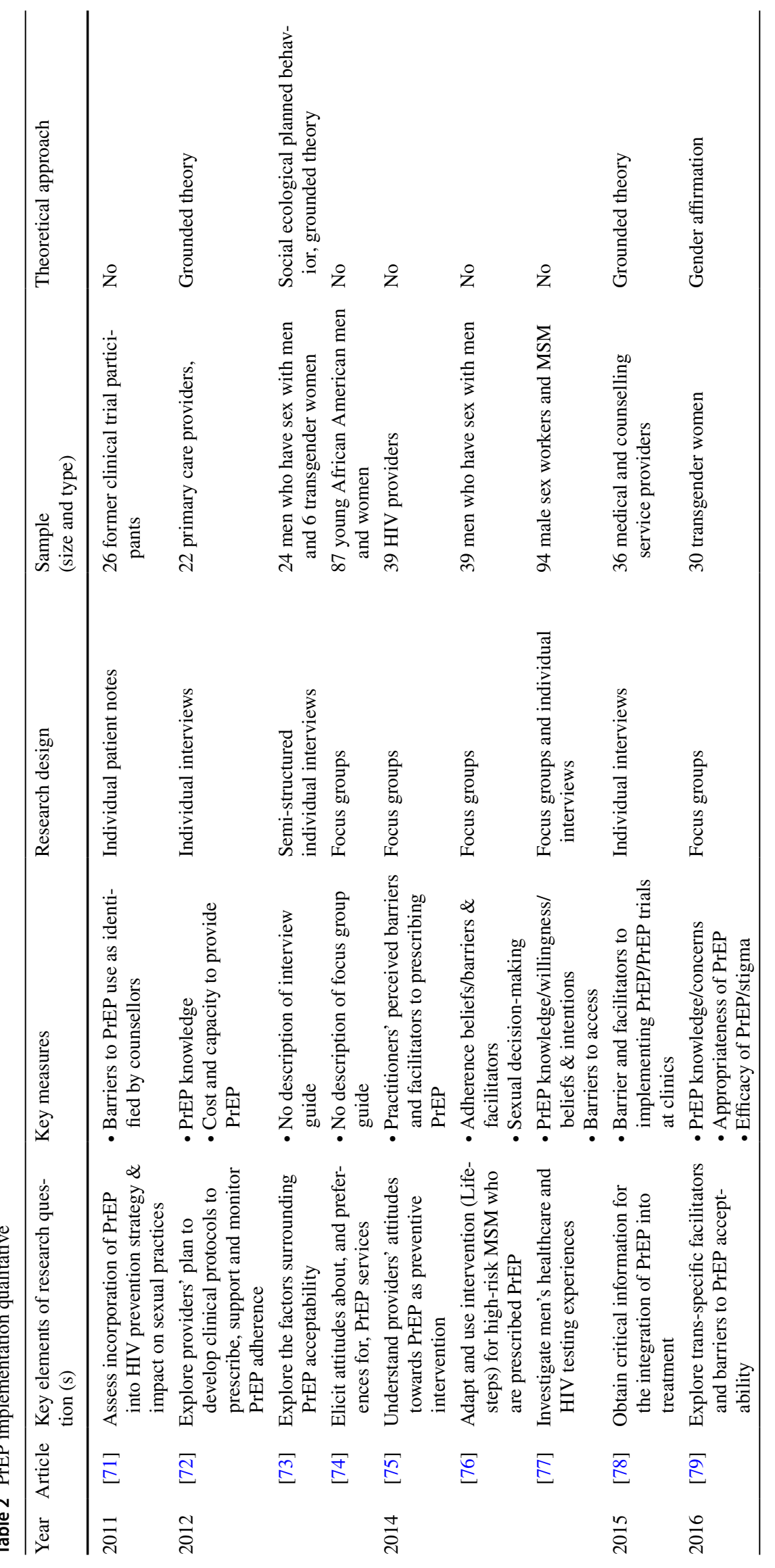




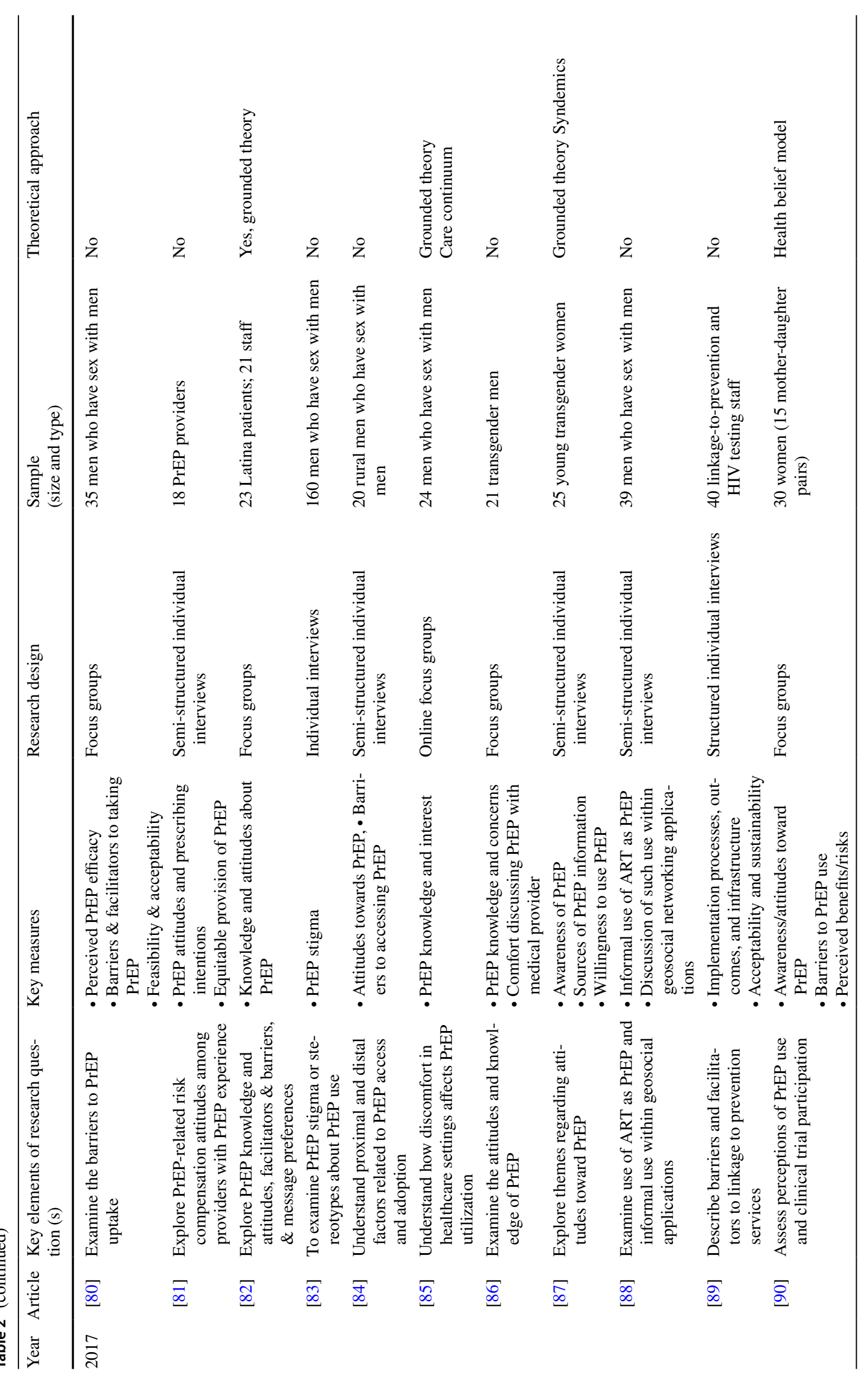




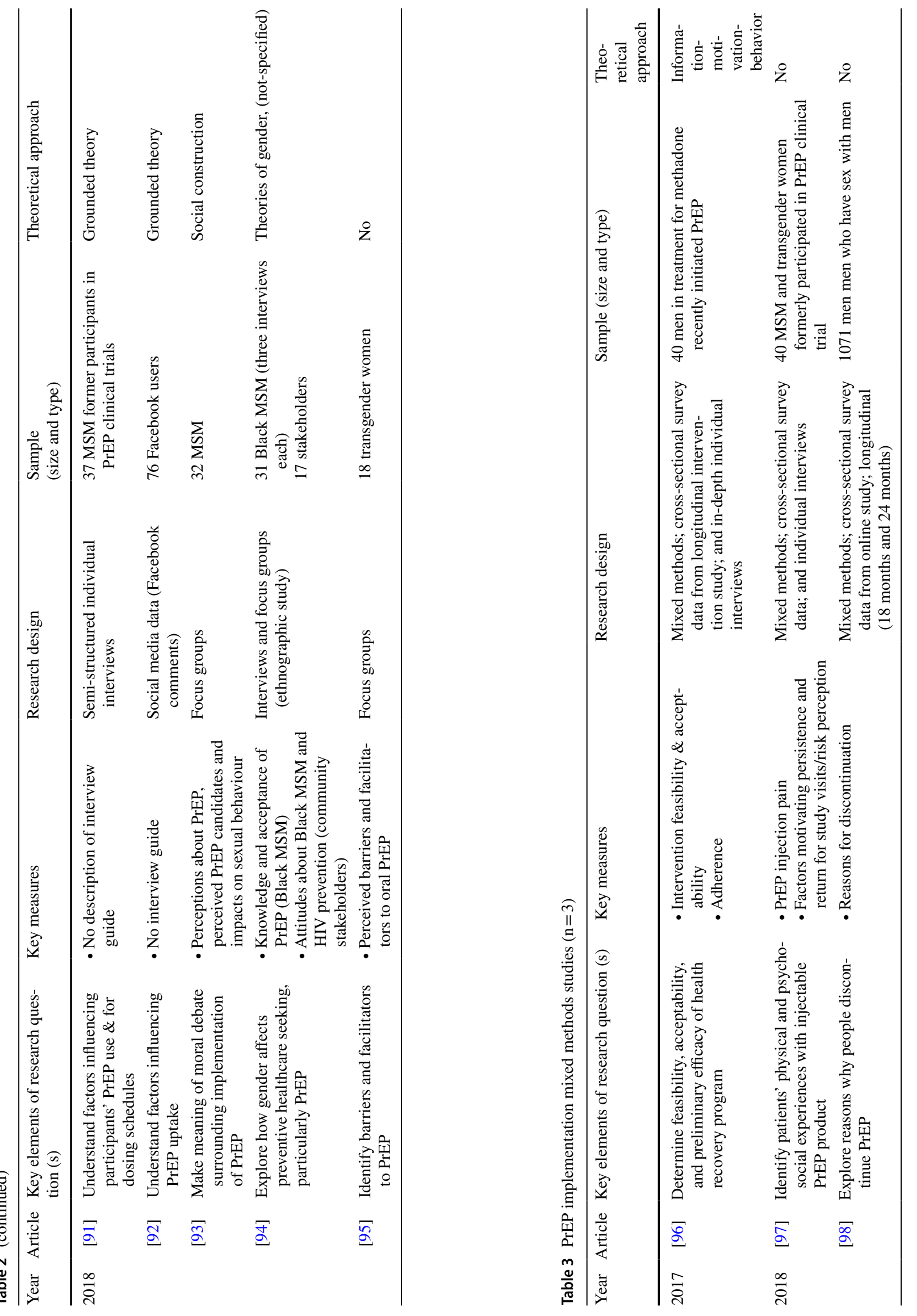


prospective and retrospective collection of administrative (clinic) data, and longitudinal study designs. One quantitative study used multiple data sources, combining clinic/ administrative data and a cross-sectional patient exit survey.

Qualitative articles used a diversity of approaches, including semi-structured individual interviews $(\mathrm{n}=10 / 25$, $40.1 \%)$ or focus groups alone $(n=11 / 25,44.0 \%)$; one of these focus groups used innovative online focus group methods. Two studies included both focus groups and individual interviews, one of which used a longitudinal approach, interviewing participants on three different occasions. The remaining qualitative studies involved qualitative analysis of patient notes and qualitative analysis of social media (Facebook) posts.

Mixed methods were the minority $(\mathrm{n}=3)$. One article used a cross-sectional survey in combination with semistructured individual interviews. The other two articles used longitudinal surveys, one in combination with semistructured individual interviews and the other with online open-ended survey questions.

\section{Sample Types and Sizes}

Of 51 quantitative studies, 31 were conducted with patients, 19 with healthcare providers, and one used data from PrEP clinics. Of 25 qualitative studies, 18 were conducted with patients, five with healthcare providers, and two included both patients and healthcare providers. All three mixed methods studies were conducted with patients.

Sample size for quantitative studies conducted with healthcare providers ranged from 35 to 9023 participants (median: 238), and sample size for quantitative studies conducted with patients ranged from 18 to 2297 participants (median: 205). Across all quantitative studies, the median sample size per year fluctuated from a low of 164 in 2014 to a high of 335 in 2016. Sample size for qualitative studies conducted with healthcare providers ranged from 18 to 39 participants (median: 36), and sample size for qualitative studies conducted with patients ranged from 18 to 160 participants (median: 31 ). Across all qualitative studies, the median sample size per year fluctuated from a low of 24 in 2012 to a high of 39 in 2014. Mixed methods sample sizes included 40 (two studies) and 1071.

The largest number of studies $(n=25 / 79,32 \%)$ with patients focused on MSM $(n=20)$ or MSM combined with transgender women $(n=5)$. Other key populations included transgender women alone $(n=4)$ and men $(n=1)$, people who use drugs $(n=3)$, and adolescents $(n=1)$. Five studies were explicitly focused on African American/Black populations, two of which were focused on Black MSM and two of which were focused on Black women. Articles focused on healthcare providers included physicians, infectious disease and other specialists, fellows, residents, in addition to physician assistants, community-based providers, clinician researchers, nurses and nurse practitioners, pharmacists, and medical students. Notably, one study surveyed health department directors.

\section{Underlying Theoretical Approach}

We identified whether or not the research questions pursued in the articles were theoretically framed, and then we identified the theory/concepts used. One-quarter $(n=12 / 51$, $24 \%)$ of quantitative articles, under half $(n=12 / 25,48 \%)$ of qualitative, and one of three mixed methods $(n=1 / 3,33 \%)$ described a conceptual framework/theoretical approach, including: grounded theory; diffusion of innovation; message framing; theory of planned behavior; purview paradox; network theory; couples interdependence theory; social cognitive theory; transtheoretical model; social ecological approach; gender affirmation; care continuum model; syndemics; health belief model; social constructionism; and information-motivation-behavior theory. One study described choosing variables based on theoretical significance without explicitly mentioning a theory while another described their own conceptual model, and yet another described including theories of gender, health, and sexuality without specifying.

\section{Discussion}

Research on PrEP implementation has been robust and consistent for the past decade. This literature has employed myriad methodologies and has succeeded in identifying important barriers that affect providers, patients, and health care systems. Studies have focused on populations and communities (e.g., MSM, African America, transgender women) most affected by HIV. In so doing, the literature has uncovered structural barriers and systemic hindrances-PrEP stigma, HIV-stigma, homophobia, transphobia, racism-affecting the most vulnerable individuals.

PrEP was approved by the FDA in 2012. It is not surprising that all articles that were included in this methodological review began to appear around 2011. Up to 2011, articles about PrEP had focused on PrEP awareness and attitudes, summaries and discussions about clinical guidelines, models to determine PrEP eligibility, editorials and descriptive commentaries-these articles were excluded from this review. Since 2012, the number of qualitative, quantitative, and mixed methods publications about PrEP implementation has risen in all three categories; with quantitative studies about patients representing the largest increase. Early studies (2012-2015) focused on factors that could facilitate access to PrEP, and thus they were more likely to be about providers' knowledge, attitudes, and PrEP acceptability as a novel 
treatment as a prevention strategy. Later studies, particularly those 2018 onwards, have focused largely on the nuances of PrEP decision-making and ongoing engagement with PrEP.

Although the majority of articles included patients and/or used quantitative study designs, the use of particular methods and study samples has differed over time. Early PrEP implementation studies were primarily qualitative, capturing the voices of patients (2011 onwards) and providers (2012 onwards). Quantitative studies selected for this review began to emerge in 2013 and continued steadily at two or more publications per year, with a total of 20 in 2018.

Qualitative research about PrEP implementation has always been in the minority. Early articles unearthed descriptive information, from both patients and providers, which grounded future quantitative research that collected more specific survey data on barriers to PrEP uptake, accessibility, and delivery. Early qualitative studies were formative and used small samples, except for three studies focused on Black MSM, and which used samples involving 87 (74), 94 (77), and 160 (83) participants. Quantitative exploratory studies began to appear in 2013-2014 and the number of publications grew steadily. More sophisticated evaluative [27], comparative [32], and associative [52] research appeared in more recent years (2016-2018). One innovation in qualitative research came about in 2017; one study [85] used an online focus group method in order to examine how discomfort in healthcare settings affects PrEP utilization.

In general, PrEP research has explored cognitive constructs (knowledge, attitude, opinions, concerns, and awareness about PrEP) that are applicable to both patient and provider research participants. Behavioral measures have included, for example, adherence to PrEP among patients and willingness to prescribe PrEP among providers. However, the majority of articles have been exploratory and cross-sectional. Therefore, there is a dearth of longitudinal articles that could capture the fast-paced changes related to PrEP implementation. For example, it is clear that, over time, both patients and providers have developed more awareness, become more knowledgeable, developed better attitudes and fewer concerns about PrEP. Nonetheless, it is not clear, from this literature, whether these changes are sustained over time or the extent to which identified changes influence patient access and adherence to PrEP. Similarly, it is unclear the extent to which cognitive changes among providers may or may not influence providers' future prescribing behaviors.

The majority of articles about barriers to PrEP implementation aimed to examine cognitive constructs and behaviors of one or another key actor-service providers or patients. Studies about patient-level barriers often have small samples and often focus on one population or another. These studies lack the power and/or demographic diversity that would allow for comparisons across different groups of individuals whose degrees of exposure to HIV might differ.
For example, the majority of studies in this review involved MSM-yet few were specific to young Black MSM (who are most exposed to HIV) or to other groups often overlooked (e.g., cisgender women). Studies that allow for comparisons across groups are highly needed at this juncture.

There is also a dearth of longitudinal designs that could illuminate trends in PrEP uptake. We identified six longitudinal studies, published in 2017-2018, one of which uses baseline data to assess the role of interprofessional collaboration (IPC) in PrEP access. Though the study uses crosssectional data, it stands out in that it shows providers of social and public health services having positive attitudes about and thus engaging in IPC with clinicians who can prescribe PrEP. The article suggests that IPC is a promising intervention that should be further studied [62]. One longitudinal article [66] explores past and current use of PrEP and it stands out for its unique contribution about reasons for discontinuation of PrEP. This structural-level quantitative study combines clinic, county, and state-level data to explore the distribution of publicly listed PrEP-providing clinics in the US and to match this distribution with need based on HIV incidence, among other factors. Nonetheless, this study involved only young MSM, and it is thus limited in terms of generalization to other populations. A more recent longitudinal study adds interesting knowledge in that it investigates associations between substance use, PrEP adherence, and the incidence of sexually transmitted infections among MSM in a PrEP clinical trial [54]. The other longitudinal study [60] contributes to knowledge about the role of stigma among a fairly large sample of $620 \mathrm{MSM}$ and transgender women. The unique contribution here is the multi-level approach that includes individual- and geospatial-level data.

The combined longitudinal survey data with geo-spatial city-level data has advanced knowledge about geographicand individual-level associations with PrEP stigma [60]. Previous articles identifying stigma, as a major factor that influence negatively PrEP uptake, were mostly qualitative and published before 2018. Two key qualitative studies involved a large sample of 160 MSM [83] and of 30 transgender women [79]. In these articles, stigma is studied in relationship to knowledge, and appropriateness of PrEP for specific populations. Articles concerning the role of stigma understandably included historically stigmatized and under-served populations; nonetheless, future research is needed to fully understand the role of PrEP-stigma specifically and how PrEP-stigma manifests in all populations in need of HIV prevention. For example, in more recent studies $[38,40]$, the authors examined the influence of alcohol and drug abuse on patients' beliefs and whether such beliefs influence PrEP uptake. These and other studies examining associations between alcohol and/or drug use and PrEP access, adherence, and discontinuation could also examine the influence of PrEP-stigma and thus further 
elucidate current understanding of stigma in the context of PrEP implementation.

Worthy of note is a longitudinal study among the few mixed methods studies in the PrEP implementation literature. We identified three mixed methods studies in 2017 and 2108. One article [96], which was framed by Information-Motivation-Behavior theory, used longitudinal data from an intervention study involving 40 men receiving methadone treatment and who recently initiated PrEP, and also semi-structured individual interviews. The authors of the other two mixed methods articles [97, 98] did not use a theoretical framework, but by using mixed data, they were best able to identify barriers to implementation and reasons for both discontinuation and re-initiation of PrEP. For these reasons, the mixed methods articles stand out among all the others.

\section{Recommendations for Future PrEP Research}

Having described how the methods used to study PrEP implementation have evolved over time, below we provide recommendations about how to improve PrEP implementation research as we move forward. These recommendations concern strategies to improve PrEP implementation research related to (1) designs and methods, (2) the need to involve practitioners in PrEP research and to address discipline shortages, (3) the need to address a lack of attention to the effect of geographic disparities in PrEP implementation; (4) the need for more robust research to address PrEP stigma, and (5) the need for conducting conceptually sound PrEP research.

\section{Longitudinal and Mixed Method Research}

This methodological review revealed few longitudinal and mixed methods studies. However, we demonstrated that longitudinal and/or mixed methods studies can contribute much to our understanding about how patients and providers may change cognitive structures over time and then change behaviors that may increase or decrease PrEP implementation. This type of longitudinal information is sorely needed so that researchers and policy makers can be best able to develop and test interventions to keep people engaged within what Nunn et al. [99] termed as the nine-step PrEP care continuum-identify individuals exposed to HIV, increase individual HIV-risk awareness, enhance PrEP awareness, facilitate PrEP access, link to PrEP care, prescribe PrEP, initiate PrEP, adhere to PrEP, and retain individuals in PrEP care. Similarly, articles combining qualitative and survey data have been best able to contextualize barriers to implementation and best explain how we might overcome them.

\section{Ethnographic and Observational Research}

We have been unable to identify more than one ethnographic or observational study that would provide details about the conditions under which patients and providers make PrEPrelated decisions, and how, in turn, different decision-making strategies might influence diverse demographic patient groups to access and adhere to PrEP. One article, reporting findings from an ethnographic approach, advances current knowledge by revealing structures, such as the healthcare system and the labor market, which alone or together may hinder PrEP uptake by systematically constraining men's access to primary providers [94]. More studies that focus on day-to-day functioning of organizations that provide PrEP services are needed in order to advance knowledge about referral-making strategies that might lead to successful access and adherence to PrEP. Such studies could also integrate policy analysis in their designs in an attempt to clarify the influence of policy guidelines on PrEP-related behaviors concerning both patients (e.g., adherence) and providers (e.g., prescribing). For example, in one article in this review [45], the authors showed that insurance status was associated with PrEP use. This important information could be more helpful if the authors had incorporated contextual data to show how insurance status influences PrEP use.

\section{Involving Practitioners in PrEP Research}

In order to help patients to access and adhere to PrEP, providers of social and public health services have a crucial role as they have the knowledge and skills to "move" patients along the PrEP continuum. Regrettably, these providers have been neglected in the current literature. Nonetheless, our research team has shown, for example, that in multivariate analysis higher interprofessional collaboration scores were associated with delivering psychoeducation about PrEP and linking patients to more services along the PrEP continuum [62]. This is an area of research with great potential to uncover specific factors related to interprofessional collaboration and which might inform future intervention for service providers. Therefore, we recommend further inquiry in this area.

\section{Geographic Disparities in PrEP Research}

Few studies $[60,66]$ were found that account for the availability of PrEP in specific geographic contexts. Because HIV risk is different within populations in myriad geographic areas, PrEP research will need to focus on these populations and special attention will be needed in order to uncover the specific socioeconomic factors that influence PrEP implementation and how these factors may differ by geographic and political contexts. This level of detail will be needed 
in order to address disparities related to PrEP access and uptake.

\section{Discipline Shortages Need to be a Focus in PrEP Research}

Only two articles $[82,94]$ contained both provider and patient data, and only one study examined data from administrators [70]. We know that structural-level barriers, such as shortage of certain professions in select geographic locations and lack of administrative supervision have an impact on patients' capacity to access PrEP. For example, it is very hard to find infectious disease physicians, those more likely to prescribe PrEP, in rural areas [100]. Similarly, there are enormous disparities concerning the presence of providers of social and public health services in rural and urban areas, and many may lack regular supervision [101]. A national survey of social workers showed that only $8 \%$ of respondents practiced in rural areas [102]. Without the help of competent providers, many patients cannot move through the PrEP continuum and will be exposed to HIV without protection. Therefore, research in this area is encouraged.

\section{PrEP Stigma Research}

Research to uncover the influence of PrEP-stigma is needed across all populations. We hypothesize that higher degrees of stigma will be found among populations historically under-represented in research (e.g., women and young people), and among racial/ethnic and sexual minorities. Though we have identified articles that examined the role of stigma, future studies ought to examine the intersectional nature of stigma and how it manifests for people facing myriad intersecting structural disadvantages, such as poverty, multiple medical issues, racism, xenophobia, and others. Moreover, since HIV exposure is higher among individuals who use drugs and alcohol [103], we recommend studies to uncover the specific needs of this population.

\section{Conceptually Sound PrEP Research}

Only 25 out of 79 articles explicitly identified a conceptual framework. The majority of these articles used individuallevel theories to guide their choices of variables. These studies did not examine either theoretically or empirically structural issues that may influence PrEP implementation. Compared to articles without conceptual frameworks, those that were framed theoretically were more useful in that they generated findings that can more readily inform the development of interventions for both patients and providers. The same theoretical approaches used to uncover barriers to PrEP can inform intervention development. Moreover, we recommend a combination of theory, longitudinal design, and mixed method approaches. Studies that used this combination allowed the authors to make stronger assertions about their findings and also about corresponding interventions that they recommended.

\section{Conclusion}

Based on our methodological review of the PrEP implementation literature, we conclude that the methods used thus far progressed since PrEP became a major HIV prevention strategy. From a preponderance of formative and descriptive small qualitative studies, we have developed larger and more predictive studies. Nonetheless, there is a paucity of longitudinal and mixed methods studies, those with the best potential to illuminate future practice and policy development regarding PrEP implementation. The majority of studies identified lack theoretical frameworks, and thus may have limitations concerning the applicability of their results. The integration of theory in health services research can improve methodology, which ultimately produces stronger research findings to inform decision-making at organizational and policy levels [104]. As we move forward, researchers will need to strive to take this information into consideration when developing and conducting studies about PrEP implementation. In so doing, we might be better able to develop interventions to break PrEP implementation barriers situated at multiple ecological domains, and thus improve PrEP access, uptake, and adherence. Future research should also shift from models of "cultural competency" to "structural competency" [105] as a new approach to address structural stigma [106] affecting the most vulnerable populations exposed to HIV.

Open Access This article is distributed under the terms of the Creative Commons Attribution 4.0 International License (http://creativecommons.org/licenses/by/4.0/), which permits unrestricted use, distribution, and reproduction in any medium, provided you give appropriate credit to the original author(s) and the source, provide a link to the Creative Commons license, and indicate if changes were made.

\section{References}

1. Centers for Disease Control and Prevention. Estimated HIV incidence and prevalence in the United States 2010-2015; 2018.

2. Grant RM, Lama JR, Anderson PL, et al. Preexposure chemoprophylaxis for HIV prevention in men who have sex with men. N Engl J Med. 2010;363(27):2587-99.

3. Donnell D, Baeten JM, Bumpus NN, et al. HIV protective efficacy and correlates of tenofovir blood concentrations in a clinical trial of PrEP for HIV prevention. J Acquir Immune Defic Syndr. 2014;66(3):340-8. 
4. Anderson PL, Glidden DV, Liu A, et al. Emtricitabine-tenofovir concentrations and pre-exposure prophylaxis efficacy in men who have sex with men. Sci Transl Med. 2012;4(151):151ra125.

5. Fitch L. Tracking global oral PrEP provision: the who, what and where of oral PrEP. HIV Research for Prevention conference (HIVR4P 2018). Madrid, Spain; 2018.

6. McCray E, Mermin J. Dear Colleagues: CDC Observes National Gay Men's HIV/AIDS Awareness Day 2018. 2018; https://www. hiv.gov/blog/dear-colleagues-cdc-observes-national-gay-men-shivaids-awareness-day-2018.

7. Pinto RM, Berringer KR, Melendez R, Mmeje O. Improving PrEP implementation through multilevel interventions: a synthesis of the literature. AIDS Behav. 2018;22(11):3681-91.

8. Proctor EK, Landsverk J, Aarons G, Chambers D, Glisson C, Mittman B. Implementation research in mental health services: an emerging science with conceptual, methodological, and training challenges. Adm Policy Ment Health. 2009;36(1):24-34.

9. Mugavero MJ, Amico KR, Horn T, Thompson MA. The state of engagement in HIV care in the United States: from cascade to continuum to control. Clin Infect Dis. 2013;57(8):1164-71.

10. Whittemore R, Knafl K. The integrative review: updated methodology. J Adv Nurs. 2005;52(5):546-53.

11. Munn Z, Stern C, Aromataris E, Lockwood C, Jordan Z. What kind of systematic review should I conduct? A proposed typology and guidance for systematic reviewers in the medical and health sciences. BMC Med Res Methodol. 2018;18(1):5.

12. Krieger N. Theories for social epidemiology in the 21st century: an ecosocial perspective. Int J Epidemiol. 2001;30(4):668-77.

13. Baeten JM, Donnell D, Ndase P, et al. Antiretroviral prophylaxis for HIV prevention in heterosexual men and women. N Engl $\mathrm{J}$ Med. 2012;367(5):399-410.

14. Thigpen MC, Kebaabetswe PM, Paxton LA, et al. Antiretroviral preexposure prophylaxis for heterosexual HIV transmission in Botswana. N Engl J Med. 2012;367(5):423-34.

15. FDA. FDA approves first medication to reduce HIV risk. Silver Spring; 2012

16. Kojima N, Davey DJ, Klausner JD. Pre-exposure prophylaxis for HIV infection and new sexually transmitted infections among men who have sex with men. AIDS. 2016;30(14):2251-2.

17. Pilkington V, Hill A, Hughes S, Nwokolo N, Pozniak A. How safe is TDF/FTC as PrEP? A systematic review and meta-analysis of the risk of adverse events in 13 randomised trials of PrEP. J Virus Erad. 2018;4(4):215-24.

18. Cypress BS. Rigor or reliability and validity in qualitative research: Perspectives, strategies, reconceptualization, and recommendations. Dimens Crit Care Nurs. 2017;36(4):253-63.

19. Strauss A, Corbin JM. Basics of qualitative research: grounded theory procedures and techniques. Thousand Oaks: Sage Publications, Inc.; 1990.

20. Puro V, Palummieri A, De Carli G, Piselli P, Ippolito G. Attitude towards antiretroviral pre-exposure prophylaxis (PrEP) prescription among HIV specialists. BMC Infect Dis. 2013;13:217.

21. Tellalian D, Maznavi K, Bredeek UF, Hardy WD. Pre-exposure prophylaxis (PrEP) for HIV infection: results of a survey of HIV healthcare providers evaluating their knowledge, attitudes, and prescribing practices. AIDS Patient Care STDs. 2013;27(10):553-9.

22. Calabrese SK, Earnshaw VA, Underhill K, Hansen NB, Dovidio JF. The impact of patient race on clinical decisions related to prescribing HIV pre-exposure prophylaxis (PrEP): assumptions about sexual risk compensation and implications for access. AIDS Behav. 2014;18(2):226-40.

23. Shaeer KM, Sherman EM, Shafiq S, Hardigan P. Exploratory survey of Florida pharmacists' experience, knowledge, and perception of HIV pre-exposure prophylaxis. J Am Pharm Assoc. 2014;54(6):610-7.
24. Blumenthal J, Jain S, Krakower D, et al. Knowledge is power! Increased provider knowledge scores regarding pre-exposure prophylaxis (PrEP) are associated with higher rates of PrEP prescription and future intent to prescribe PrEP. AIDS Behav. 2015;19(5):802-10.

25. Castel AD, Feaster DJ, Tang W, et al. Understanding HIV Care Provider Attitudes Regarding Intentions to Prescribe PrEP. J Acquir Immune Defic Syndr. 2015;70(5):520-8.

26. Krakower DS, Oldenburg CE, Mitty JA, et al. Knowledge, beliefs and practices regarding antiretroviral medications for hiv prevention: results from a survey of healthcare providers in New England. PLoS ONE. 2015;10(7):e0132398.

27. Chan PA, Mena L, Patel R, et al. Retention in care outcomes for HIV pre-exposure prophylaxis implementation programmes among men who have sex with men in three US cities. J Int AIDS Soc. 2016;19(1):20903.

28. Hakre S, Blaylock JM, Dawson P, et al. Knowledge, attitudes, and beliefs about HIV pre-exposure prophylaxis among US air force health care providers. Med. 2016;95(32):e4511.

29. Krakower DS, Maloney KM, Grasso C, Melbourne K, Mayer KH. Primary care clinicians' experiences prescribing HIV pre-exposure prophylaxis at a specialized community health centre in Boston: lessons from early adopters. J Int AIDS Soc. 2016;19(1):21165.

30. Lelutiu-Weinberger C, Golub SA. Enhancing PrEP access for black and latino men who have sex with men. J Acquir Immune Defic Syndr. 2016;73(5):547-55.

31. Smith DK, Mendoza MC, Stryker JE, Rose CE. PrEP Awareness and attitudes in a national survey of primary care clinicians in the United States, 2009-2015. PLoS ONE. 2016;11(6): e0156592.

32. Biello KB, Oldenburg CE, Mitty JA, et al. The "safe sex" conundrum: anticipated stigma from sexual partners as a barrier to PrEP use among substance using MSM engaging in transactional sex. AIDS Behav. 2017;21(1):300-6.

33. Blackstock OJ, Moore BA, Berkenblit GV, et al. A cross-sectional online survey of HIV pre-exposure prophylaxis adoption among primary care physicians. J Gen Intern Med. 2017;32(1):62-70.

34. Doblecki-Lewis S, Liu A, Feaster D, et al. Healthcare access and PrEP continuation in San Francisco and Miami after the US PrEP Demo Project. J Acquir Immune Defic Syndr. 2017;74(5):531-8.

35. Eaton LA, Kalichman SC, Price D, Finneran S, Allen A, Maksut J. Stigma and conspiracy beliefs related to pre-exposure prophylaxis (PrEP) and interest in using PrEP among Black and White men and transgender women who have sex with men. AIDS Behav. 2017;21(5):1236-46.

36. Edelman EJ, Moore BA, Calabrese SK, et al. Primary care physicians' willingness to prescribe HIV pre-exposure prophylaxis for people who inject drugs. AIDS Behav. 2017;21(4):1025-33.

37. Garfinkel DB, Alexander KA, McDonald-Mosley R, Willie TC, Decker MR. Predictors of HIV-related risk perception and PrEP acceptability among young adult female family planning patients. AIDS Care. 2017;29(6):751-8.

38. Golub SA, Gamarel KE, Lelutiu-Weinberger C. The importance of sexual history taking for PrEP comprehension among young people of color. AIDS Behav. 2017;21(5):1315-24.

39. Holloway IW, Tan D, Gildner JL, et al. Facilitators and barriers to pre-exposure prophylaxis willingness among young men who have sex with men who use geosocial networking applications in California. AIDS Patient Care STDs. 2017;31(12):517-27.

40. Kalichman SC, Eaton L. Alcohol-antiretroviral interactive toxicity beliefs as a potential barrier to HIV pre-exposure prophylaxis among men who have sex with men. J Int AIDS Soc. 2017;20(1):21534.

41. Kuhns LM, Hotton AL, Schneider J, Garofalo R, Fujimoto K. Use of pre-exposure prophylaxis (PrEP) in young men who have 
sex with men is associated with race, sexual risk behavior, and peer network size. AIDS Behav. 2017;21(5):1376-82.

42. Marks SJ, Merchant RC, Clark MA, et al. Potential healthcare insurance and provider barriers to pre-exposure prophylaxis utilization among young men who have sex with men. AIDS Patient Care STDs. 2017;31(11):470-8.

43. Mullins TLK, Zimet G, Lally M, Xu J, Thornton S, Kahn JA. HIV care providers' intentions to prescribe and actual prescription of pre-exposure prophylaxis to at-risk adolescents and adults. AIDS Patient Care STDs. 2017;31(12):504-16.

44. Parisi D, Warren B, Leung SJ, et al. A multicomponent approach to evaluating a pre-exposure prophylaxis (PrEP) implementation program in five agencies in New York. J Assoc Nurses AIDS Care. 2018;29(1):10-9.

45. Patel RR, Mena L, Nunn A, et al. Impact of insurance coverage on utilization of pre-exposure prophylaxis for HIV prevention. PLoS ONE. 2017;12(5):e0178737.

46. Patel RR, Harrison LC, Patel VV, et al. HIV pre-exposure prophylaxis programs incorporating social applications can reach at-risk men who have sex with men for successful linkage to care in Missouri, USA. J Assoc Nurses AIDS Care. 2017;28(3):428-30.

47. Petroll AE, Walsh JL, Owczarzak JL, McAuliffe TL, Bogart LM, Kelly JA. PrEP awareness, familiarity, comfort, and prescribing experience among US primary care providers and HIV specialists. AIDS Behav. 2017;21(5):1256-67.

48. Raifman JR, Flynn C, German D. Healthcare provider contact and pre-exposure prophylaxis in Baltimore men who have sex with men. Am J Prev Med. 2017;52(1):55-63.

49. Walters SM, Reilly KH, Neaigus A, Braunstein S. Awareness of pre-exposure prophylaxis (PrEP) among women who inject drugs in NYC: the importance of networks and syringe exchange programs for HIV prevention. Harm Reduct J. 2017;14(1):40.

50. Blaylock JM, Hakre S, Decker CF, et al. HIV PrEP in the military: experience at a tertiary care military medical center. Mil Med. 2018;183(Suppl_1):445-9.

51. Broekhuis JM, Scarsi KK, Sayles HR, et al. Midwest pharmacists' familiarity, experience, and willingness to provide pre-exposure prophylaxis (PrEP) for HIV. PLoS ONE. 2018;13(11):e0207372.

52. Calabrese SK, Earnshaw VA, Krakower DS, et al. A closer look at racism and heterosexism in medical students' clinical decisionmaking related to HIV pre-exposure prophylaxis (PrEP): implications for PrEP education. AIDS Behav. 2018;22(4):1122-38.

53. Guinness RR, Volk JE, Hurley LB, Tobias TT, Marcus JL. Lowintensity outreach to increase uptake of HIV preexposure prophylaxis among patients with sexually transmitted infections. AIDS Behav. 2018;23(2):544-7.

54. Hoenigl M, Jain S, Moore D, et al. Substance use and adherence to HIV preexposure prophylaxis for men who have sex with men. Emerg Infect Dis. 2018;24(12):2292.

55. John SA, Starks TJ, Rendina HJ, Grov C, Parsons JT. Should I convince my partner to go on pre-exposure prophylaxis (PrEP)? the role of personal and relationship factors on PrEP-related social control among gay and bisexual men. AIDS Behav. 2018;22(4):1239-52.

56. Kwakwa HA, Bessias S, Sturgis D, et al. Engaging United States black communities in HIV pre-exposure prophylaxis: analysis of a PrEP engagement cascade. J Natl Med Assoc. 2018;110(5):480-5.

57. Lankowski AJ, Bien-Gund CH, Patel VV, Felsen UR, Silvera $\mathrm{R}$, Blackstock OJ. PrEP in the real world: predictors of 6-month retention in a diverse urban cohort. AIDS Behav. 2018;23(7):1797-802.

58. Meyerson BE, Dinh PC Jr, Agley JD, et al. Predicting pharmacist dispensing practices and comfort related to pre-exposure prophylaxis for HIV prevention (PrEP). AIDS Behav. 2019;23(7):1925-38.

59. Morgan E, Ryan DT, Newcomb ME, Mustanski B. High rate of discontinuation may diminish PrEP coverage among young men who have sex with men. AIDS Behav. 2018;22(11):3645-8.

60. Mustanski B, Ryan DT, Hayford C, Phillips G 2nd, Newcomb ME, Smith JD. Geographic and individual associations with PrEP stigma: results from the RADAR cohort of diverse young men who have sex with men and transgender women. AIDS Behav. 2018;22(9):3044-56.

61. Okoro O, Hillman L. HIV pre-exposure prophylaxis: exploring the potential for expanding the role of pharmacists in public health. J Am Pharm Assoc. 2018;58(4):412-20 (e413).

62. Pinto RM, Witte SS, Filippone P, Choi CJ, Walls M. Interprofessional collaboration and on-the-job training improve access to HIV testing, HIV primary care, and pre-exposure prophylaxis (PrEP). AIDS Educ Prev. 2018;30(6):474-89.

63. Rolle CP, Rosenberg ES, Siegler AJ, et al. Challenges in translating PrEP interest into uptake in an observational study of young black MSM. J Acquir Immune Defic Syndr. 2017;76(3):250-8.

64. Roth AM, Aumaier BL, Felsher MA, et al. An exploration of factors impacting preexposure prophylaxis eligibility and access among syringe exchange users. Sex Trans Dis. 2018;45(4):217-21.

65. Shrestha R, Altice FL, Karki P, Copenhaver MM. Integrated bio-behavioral approach to improve adherence to pre-exposure prophylaxis and reduce HIV risk in people who use drugs: a pilot feasibility study. AIDS Behav. 2018;22(8):2640-9.

66. Siegler AJ, Bratcher A, Weiss KM, Mouhanna F, Ahlschlager L, Sullivan PS. Location location location: an exploration of disparities in access to publicly listed pre-exposure prophylaxis clinics in the United States. Ann Epidemiol. 2018;28(12):858-64.

67. Tekeste M, Hull S, Dovidio JF, et al. Differences in medical mistrust between black and white women: implications for patient-provider communication about PrEP. AIDS Behav. 2018;23(7):1737-48.

68. van Epps P, Maier M, Lund B, et al. Medication adherence in a nationwide cohort of veterans initiating pre-exposure prophylaxis (PrEP) to prevent HIV infection. J Acquir Immune Defic Syndr. 2018;77(3):272-8.

69. Willie TC, Stockman JK, Overstreet NM, Kershaw TS. Examining the impact of intimate partner violence type and timing on pre-exposure prophylaxis awareness, interest, and coercion. AIDS Behav. 2018;22(4):1190-200.

70. Zhang HL, Rhea SK, Hurt CB, et al. HIV preexposure prophylaxis implementation at local health departments: a statewide assessment of activities and barriers. J Acquir Immune Defic Syndr. 2018;77(1):72-7.

71. Hojilla CJ, Koester KA, Cohen SE, et al. Sexual behavior, risk compensation, and HIV prevention strategies among participants in the San Francisco PrEP demonstration project: a qualitative analysis of counseling notes. AIDS Behav. 2016;20(7):1461-9.

72. Arnold EA, Hazelton P, Lane T, et al. A qualitative study of provider thoughts on implementing pre-exposure prophylaxis (PrEP) in clinical settings to prevent HIV infection. PLoS ONE. 2012;7(7):e40603.

73. Galindo GR, Walker JJ, Hazelton P, et al. Community member perspectives from transgender women and men who have sex with men on pre-exposure prophylaxis as an HIV prevention strategy: implications for implementation. Implement Sci. 2012;7:116.

74. Smith DK, Toledo L, Smith DJ, Adams MA, Rothenberg R. Attitudes and program preferences of African-American urban young adults about pre-exposure prophylaxis (PrEP). AIDS Educ Prev. 2012;24(5):408-21. 
75. Krakower D, Ware N, Mitty JA, Maloney K, Mayer KH. HIV providers' perceived barriers and facilitators to implementing pre-exposure prophylaxis in care settings: a qualitative study. AIDS Behav. 2014;18(9):1712-21.

76. Taylor SW, Mayer KH, Elsesser SM, Mimiaga MJ, O'Cleirigh C, Safren SA. Optimizing content for pre-exposure prophylaxis (PrEP) counseling for men who have sex with men: perspectives of PrEP users and high-risk PrEP naïve men. AIDS Behav. 2014;18(5):871-9.

77. Underhill K, Morrow KM, Colleran CM, et al. Access to healthcare, HIV/STI testing, and preferred pre-exposure prophylaxis providers among men who have sex with men and men who engage in street-based sex work in the US. PLoS ONE. 2014;9(11):e112425.

78. Spector AY, Remien RH, Tross S. PrEP in substance abuse treatment: a qualitative study of treatment provider perspectives. Subst Abuse Treat Prev Policy. 2015;10:1.

79. Sevelius JM, Keatley J, Calma N, Arnold E. 'I am not a man': trans-specific barriers and facilitators to PrEP acceptability among transgender women. Glob Public Health. 2016;11(7-8):1060-75.

80. Cahill S, Taylor SW, Elsesser SA, Mena L, Hickson D, Mayer KH. Stigma, medical mistrust, and perceived racism may affect PrEP awareness and uptake in black compared to white gay and bisexual men in Jackson, Mississippi and Boston, Massachusetts. AIDS Care. 2017;29(11):1351-8.

81. Calabrese SK, Magnus M, Mayer KH, et al. "Support your client at the space that they're in": HIV pre-exposure prophylaxis (PrEP) prescribers' perspectives on PrEP-related risk compensation. AIDS Patient Care STDs. 2017;31(4):196-204.

82. Collier KL, Colarossi LG, Sanders K. Raising awareness of pre-exposure prophylaxis (PrEP) among women in New York City: community and provider perspectives. J Health Commun. 2017;22(3):183-9.

83. Golub SA, Gamarel KE, Surace A. Demographic differences in PrEP-related stereotypes: implications for implementation. AIDS Behav. 2017;21(5):1229-35.

84. Hubach RD, Currin JM, Sanders CA, et al. Barriers to access and adoption of pre-exposure prophylaxis for the prevention of HIV among men who have sex with men (MSM) in a relatively rural state. AIDS Educ Prev. 2017;29(4):315-29.

85. Maloney KM, Krakower DS, Ziobro D, Rosenberger JG, Novak $\mathrm{D}$, Mayer KH. Culturally competent sexual healthcare as a prerequisite for obtaining preexposure prophylaxis: findings from a qualitative study. LGBT Health. 2017;4(4):310-4.

86. Rowniak S, Ong-Flaherty C, Selix N, Kowell N. Attitudes, beliefs, and barriers to PrEP among trans men. AIDS Educ Prev. 2017;29(4):302-14.

87. Wood SM, Lee S, Barg FK, Castillo M, Dowshen N. Young transgender women's attitudes toward HIV pre-exposure prophylaxis. J Adolesc Health. 2017;60(5):549-55.

88. Buttram ME, Kurtz SP. Representations of Pre-exposure prophylaxis, informal antiretroviral medication use for HIV prevention, and diversion on geosocial networking apps among men who have sex with men. AIDS Behav. 2018;23(7):1790-6.

89. Doll M, Fortenberry JD, Roseland D, McAuliff K, Wilson CM, Boyer CB. Linking HIV-negative youth to prevention services in 12 U.S. Cities: barriers and facilitators to implementing the HIV prevention continuum. J Adolesc Health. 2018;62(4):424-33.

90. Fletcher FE, Fisher C, Buchberg MK, et al. "Where did this [PrEP] come rrom?" African american mother/daughter perceptions related to adolescent preexposure prophylaxis (PrEP) utilization and clinical trial participation. J Empir Res Hum Res Ethics. 2018;13(2):173-84.
91. Franks J, Hirsch-Moverman Y, Loquere AS Jr, et al. Sex, PrEP, and stigma: experiences with HIV pre-exposure prophylaxis among New York City MSM participating in the HPTN 067/ ADAPT Study. AIDS Behav. 2017;22(4):1139-49.

92. Hill BS, Patel VV, Haughton LJ, Blackstock OJ. Leveraging social media to explore black women's perspectives on HIV pre-exposure prophylaxis. J Assoc Nurses AIDS Care. 2018;29(1):107-14.

93. Pawson M, Grov C. 'It's just an excuse to slut around': gay and bisexual mens' constructions of HIV pre-exposure prophylaxis (PrEP) as a social problem. Sociol Health Illn. 2018;40(8):1391-403.

94. Philbin MM, Parker CM, Parker RG, Wilson PA, Garcia J, Hirsch JS. Gendered social institutions and preventive healthcare seeking for black men who have sex with men: the promise of biomedical HIV prevention. Arch Sex Behav. 2018;47(7):2091-100.

95. Rael CT, Martinez M, Giguere R, et al. Barriers and facilitators to oral PrEP use among transgender women in New York city. AIDS Behav. 2018;22(11):3627-36.

96. Shrestha R, Karki P, Huedo-Medina TB, Copenhaver M. Intent to use preexposure prophylaxis (PrEP), HIV risk behaviors, and self-report neurocognitive symptoms by high-risk drug users: a mediation analysis. J Assoc Nurses AIDS Care. 2017;28(4):612-21.

97. Meyers K, Rodriguez K, Brill AL, et al. Lessons for patient education around long-acting injectable PrEP: findings from a mixed-method study of Phase II trial participants. AIDS Behav. 2018;22(4):1209-16.

98. Whitfield THF, John SA, Rendina HJ, Grov C, Parsons JT. Why I quit pre-exposure prophylaxis (PrEP)? A mixed-method study exploring reasons for PrEP discontinuation and potential re-initiation among gay and bisexual men. AIDS Behav. 2018;22(11):3566-75.

99. Nunn AS, Brinkley-Rubinstein L, Oldenburg CE, et al. Defining the HIV pre-exposure prophylaxis care continuum. AIDS. 2017;31(5):731-4.

100. Pellowski JA. Barriers to care for rural people living with HIV: a review of domestic research and health care models. J Assoc Nurses AIDS Care. 2013;24(5):422-37.

101. National Association of Social Workers (NASW). Rural social work. 2002. https://www.socialworkers.org/assets/secured/ documents/da/da2010/referred/Rural\%20Social\%20work.pdf.

102. Salsberg E, Quigley L, Acquaviva K, Wyche K \& Sliwa S. New social workers: Results of the nationwide survey of 2017 social work graduates, The National Social Work Workforce Study. The George Washington University Health Workforce Institute; 2018. Retrieved from: https://www.socialworkers.org/LinkClick. aspx?fileticket $=$ C0r5P1zkMbQ\%3D\&portalid $=0$.

103. Baliunas D, Rehm J, Irving H, Shuper P. Alcohol consumption and risk of incident human immunodeficiency virus infection: a meta-analysis. Int J Public Health. 2010;55(3):159-66.

104. Brazil K, Ozer E, Cloutier MM, Levine R, Stryer D. From theory to practice: improving the impact of health services research. BMC Health Services Res. 2005;5:1.

105. Metzl JM, Hansen H. Structural competency: theorizing a new medical engagement with stigma and inequality. Soc Sci Med. 2014;103:126-33.

106. Hatzenbuehler ML, Link BG. Introduction to the special issue on structural stigma and health. Soc Sci Med. 2014;103:1-6.

Publisher's Note Springer Nature remains neutral with regard to jurisdictional claims in published maps and institutional affiliations. 